\title{
Cooperativity of Negative Autoregulation Confers Increased Mutational Robustness
}

\author{
David C. Marciano, ${ }^{1}$ Rhonald C. Lua, ${ }^{1}$ Christophe Herman, ${ }^{1}$ and Olivier Lichtarge ${ }^{1,2,3, *}$ \\ ${ }^{1}$ Department of Molecular and Human Genetics, Baylor College of Medicine, Houston, Texas 77030, USA \\ ${ }^{2}$ Verna and Marrs McLean Department of Biochemistry and Molecular Biology, Baylor College of Medicine, \\ Houston, Texas 77030, USA \\ ${ }^{3}$ Computational and Integrative Biomedical Research Center, Baylor College of Medicine, Houston, Texas 77030, USA
}

(Received 24 June 2015; revised manuscript received 11 March 2016; published 22 June 2016)

\begin{abstract}
Negative autoregulation is universally found across organisms. In the bacterium Escherichia coli, transcription factors often repress their own expression to form a negative feedback network motif that enables robustness to changes in biochemical parameters. Here we present a simple phenomenological model of a negative feedback transcription factor repressing both itself and another target gene. The strength of the negative feedback is characterized by three parameters: the cooperativity in self-repression, the maximal expression rate of the transcription factor, and the apparent dissociation constant of the transcription factor binding to its own promoter. Analysis of the model shows that the target gene levels are robust to mutations in the transcription factor, and that the robustness improves as the degree of cooperativity in self-repression increases. The prediction is tested in the LexA transcriptional network of $E$. coli by altering cooperativity in self-repression and promoter strength. Indeed, we find robustness is correlated with the former. Considering the proposed importance of gene regulation in speciation, parameters governing a transcription factor's robustness to mutation may have significant influence on a cell or organism's capacity to evolve.
\end{abstract}

DOI: 10.1103/PhysRevLett.116.258104

Most biological systems are known to be robust to environmental variations, cell-to-cell variations and mutational perturbations [1,2]. Robustness contributes to the survival of the organism, and may also allow for the accumulation of phenotypically silent genetic variation [3-7], which, when exposed by a major perturbation or stress, can be a substrate for selection [1].

A network-level mechanism that can contribute to robustness of an organism is a negative feedback loop or autoregulation. Negative autoregulation (NAR) in gene regulatory networks is universally found across organisms. In $E$. coli, the NAR network motif is exhibited in about $40 \%$ of known transcription factors (TF) [8]. NAR may have arisen in evolution to speed the response time of gene circuits [9-11], to increase the stability of gene expression against low-frequency noise $[12,13]$, and to linearize or increase the input dynamic range of the gene's input-output response curve $[8,14]$. The latter two functions of NAR can be viewed as providing gene expression stability against transient perturbations in biochemical parameters.

Only recently have experiments been devised showing that NAR buffers against the effect of mutations. In yeast, NAR has been shown to stabilize expression levels of the ROX1 TF as a result of natural genetic variations across strains [15],

Published by the American Physical Society under the terms of the Creative Commons Attribution 3.0 License. Further distribution of this work must maintain attribution to the author(s) and the published article's title, journal citation, and DOI. suggesting that NAR does confer robustness to mutations. In the mammalian visual system, genetic perturbations that significantly alter the deactivation of photoexcited rhodopsin in rod photoreceptor cells do not much alter the amplitude of single-photon responses [16]. However, the same perturbations, when crossed into a line wherein calcium-mediated negative feedback is absent, produced larger alterations in single-photon responses, showing that negative feedback enables a more robust, stereotyped response that promotes reliable signal transmission through the visual pathway. In more recent work in bacteria, NAR was shown to impart robustness to TF mutations and stabilize expression levels [17]. However, it is an open question as to which components of NAR can influence mutational robustness.

Here we examine a model of a gene circuit with a TF, under NAR, that regulates the expression of a target gene (Fig. 1). In this model, negative regulation of protein production by a TF is specified by a Hill function with parameters that can be altered by mutation. We show that the steady-state level of the target gene product is robust or stable against variations in either the TF degradation rate $\left(\beta_{r}\right)$ or strength of binding between the TF and the promoters $\left(K_{r}\right.$ and $\left.K_{g}\right)$ when the TF is under NAR, compared with no feedback. We also predict and experimentally confirm that this robustness improves as the degree of cooperativity (Hill coefficient, $h_{r}$ ) of the TF regulation of its own expression increases. The results suggest that a selective pressure for robustness may drive the evolution of negative feedback loops in transcription networks. 


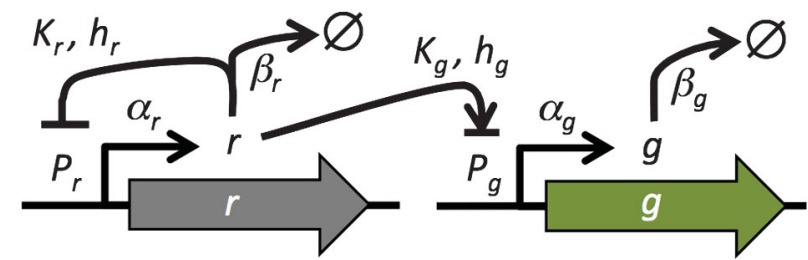

FIG. 1. Schematic of the genetic circuit containing the negatively autoregulated transcription factor (gray arrow) and target gene (green arrow). The TF represses its own promoter $\left(P_{r}\right)$ and either represses or activates the promoter of the target gene $\left(P_{g}\right)$. Slashed O sign denotes removal of gene product. Biochemical interpretations of the symbols are given in Table S.1 of the Supplemental Material [18].

Conditions satisfied in the steady-state by $T F$ and target gene levels. - The steady-state levels of the TF $(r)$ and the target gene $(g)$ are reached when protein production is balanced by protein degradation (rates $\beta_{r}$ and $\beta_{g}$ ). With negative feedback present,

$$
\beta_{r} r=\frac{\alpha_{r}}{1+\left(r / K_{r}\right)^{h_{r}}} .
$$

On the right-hand side of this equation, the repression of protein production by the TF is modeled as a Hill function [35]. Without feedback (setting $h_{r}=0$ ), we have $\beta_{r} r=\alpha_{r} / 2$. The Hill coefficient $h_{r}$ suggests the degree of cooperativity in the regulation of gene expression. The Hill repression coefficient $K_{r}$ is the TF concentration at which the protein production rate is reduced to half the maximum (rate $\alpha_{r}$ ).

The dependence of the target gene concentration on the repressor concentration is the same with or without feedback

$$
\beta_{g} g=\alpha_{g} G\left(r / K_{g}\right) .
$$

The function $G(x)$ may take the form of a Hill function $[35,36]$

$$
G\left(r / K_{g}\right)=\left[1+\left(r / K_{g}\right)^{h_{g}}\right]^{-1},
$$

where $h_{g}>0$ corresponds to repression, and $h_{g}<0$ corresponds to activation.

Target gene expression becomes less sensitive to changes in degradation rate $\tilde{\beta}_{r}$ and repressor binding $\hat{K}_{r}$ as the Hill coefficient $h_{r}$ increases.-In order to examine the effect of varying the degradation rate $\beta_{r}$ while all other parameters are held fixed, we use dimensionless variables and put $\tilde{r}=r / K_{r}, \tilde{\beta}_{r}=\left[\beta_{r} /\left(\alpha_{r} / K_{r}\right)\right]$, $g^{\prime}=\left[g /\left(\alpha_{g} / \beta_{g}\right)\right]$. With negative feedback present,

$$
\tilde{r}=\frac{1 / \tilde{\beta}_{r}}{1+\tilde{r}^{h_{r}}} .
$$

Without feedback, we have $\tilde{r}=1 / 2 \tilde{\beta}_{r}$. The dependence of the target gene concentration on the repressor is the same with or without feedback:

$$
g^{\prime}=G\left(\frac{\tilde{r}}{K_{g} / K_{r}}\right) .
$$

In order to examine the effect of varying the repression coefficient $K_{r}$, we put $\hat{r}=\left[r /\left(\alpha_{r} / \beta_{r}\right)\right], \hat{K}_{r}=\left[K_{r} /\left(\alpha_{r} / \beta_{r}\right)\right]$, $\hat{K}_{g}=\left[K_{g} /\left(\alpha_{r} / \beta_{r}\right)\right], g^{\prime}=\left[g /\left(\alpha_{g} / \beta_{g}\right)\right]$. With negative feedback present,

$$
\hat{r}=\left[1+\left(\hat{r} / \hat{K}_{r}\right)^{h_{r}}\right]^{-1} .
$$

Without feedback, we have $\hat{r}=1 / 2$. The dependence of the target gene concentration on the repressor is the same with or without feedback:

$$
g^{\prime}=G\left(\hat{r} / \hat{K}_{g}\right) .
$$

To show that the functional dependence of $g^{\prime}$ on $\hat{K}_{r}$ is the same as that of $g^{\prime}$ with $\tilde{\beta}_{r}$, multiply both sides of Eq. (6) by $1 / \hat{K}_{r}$,

$$
\hat{r} / \hat{K}_{r}=\frac{1 / \hat{K}_{r}}{1+\left(\hat{r} / \hat{K}_{r}\right)^{h_{r}}} .
$$

Using $\hat{r} / \hat{K}_{r}=\tilde{r}$, the preceding equations become

$$
\tilde{r}=\frac{1 / \hat{K}_{r}}{1+\tilde{r}^{h_{r}}}, \quad g^{\prime}=G\left(\frac{\hat{K}_{r} \tilde{r}}{\hat{K}_{g}}\right)=G\left(\frac{\tilde{r}}{K_{g} / K_{r}}\right),
$$

which are identical to Eqs. (4) and (5) if $\hat{K}_{r}$ is identified as $\tilde{\beta}_{r}$ and $K_{g} / K_{r}$ is constant.

Figure 2 plots the target gene concentration $g^{\prime}$ against $\tilde{\beta}_{r}$ at several values of $h_{r}$, where $g^{\prime}$ is calculated numerically from Eqs. (4), (5), and (3). The exact same set of curves are
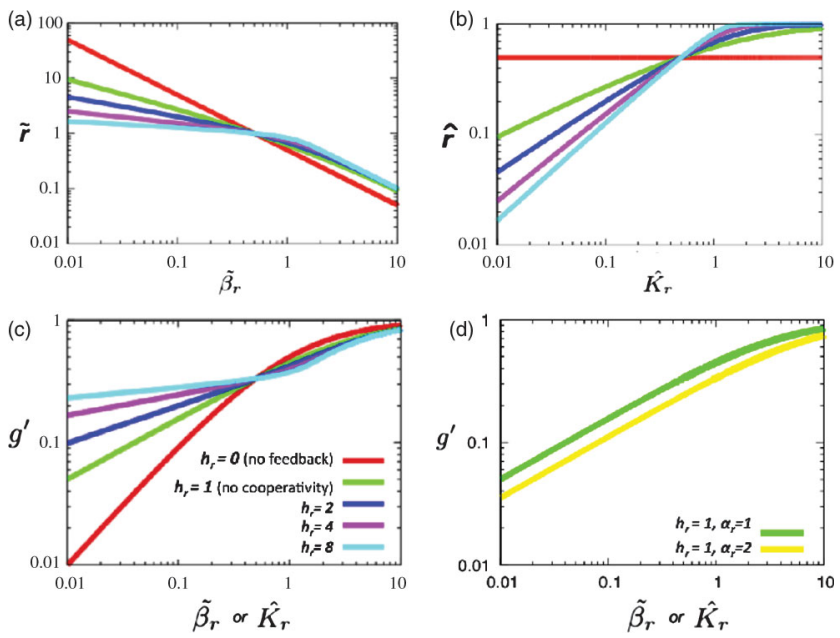

FIG. 2. Variation of $g$ and $r$ with $\beta_{r}, K_{r}$, and $h_{r}$. (a) TF concentration $\tilde{r}$ against TF degradation rate $\left(\tilde{\beta}_{r}\right)$ for different values of the Hill coefficient $h_{r}$. (b) TF concentration $\hat{r}$ against the Hill repression coefficient $\left(\hat{K}_{r}\right)$ for different values of the Hill coefficient $h_{r}$. (c) Normalized target gene concentration $g^{\prime}$ against $\tilde{\beta}_{r}$ or $\hat{K}_{r}$ for different values of the Hill coefficient $h_{r}$, showing that $g^{\prime}$ becomes more robust to TF mutations manifested as variations in either $\tilde{\beta}_{r}$ or $\hat{K}_{r}$ as $h_{r}$ increases. $g^{\prime}$ is calculated using Eq. (3) with $h_{g}=1$ (repression), $K_{g}=0.5 K_{r}$. The slope at the intersection of the curves is $2\left[3\left(1+h_{r} / 2\right)\right]^{-1}$ (see Eq. S.8, [18]). (d) $g$ decreases when $\alpha_{r}$ increases. The new values lie on a new curve that is shifted to the right. 
obtained when $g^{\prime}$ is plotted against $\hat{K}_{r}$. The calculation of the parameter sensitivity coefficients, which give the slope of these curves and which quantify robustness, are presented in the Supplemental Material [18]. Figure 2(d) also demonstrates the effect of increasing $\alpha_{r}$ on $g^{\prime}$. The new values lie on a curve shifted to the right, which lowers $g^{\prime}$, but does not decrease the parameter sensitivity coefficient and the coefficient of variation. These results show that target gene expression becomes less sensitive to perturbations in either degradation rate of the TF or its affinity to the promoter as the strength of negative feedback increases through $h_{r}$.

Experimental validation.-We used the E. coli LexA repressor of the SOS DNA damage response as a model system because it displays negative feedback by repression of its own promoter [37]. However, in order to characterize the lexA promoter ( $P_{\text {lexA }}$ and variants), we needed to break the negative feedback loop to gain control over LexA expression, determine $P_{\text {lexA }}$ activity over a range of LexA protein levels and fit the Hill equation. Replacing the lexA promoter on the chromosome with an anhydrotetracycline (ATc)-inducible tetA promoter $\left(P_{\text {tetA }}\right)$ facilitates control over LexA levels [Fig. 3(a)]. Dose-dependent transcription of lexA is inferred by measuring the fluorescence of the co-transcribed red fluorescent protein variant, mCherry (Fig. S.3, [18]). A plasmid-encoded $P_{l e x A}$ is reintroduced as a fusion to a cyan fluorescent protein variant, cerulean [Fig. 3(a)]. $P_{\text {lexA }}$ has two SOS boxes and, although not experimentally quantified, it has been reported to have moderate cooperativity $[17,36,38]$. By adding more SOS boxes $\left(P_{\text {lexA-SOS3 }}\right.$ and $\left.P_{\text {lexA-SOS5 }}\right)$, we expected to increase the cooperativity. A completely different promoter with 0 SOS boxes $\left(P_{t r c}\right)$ is a control system that is not repressed by LexA and should be unresponsive to changes in LexA levels. By deconstructing the lexA gene circuit into a simpler repression system we can experimentally measure parameters of $P_{l e x A}$ and variants thereof.

To estimate $\alpha_{r}$, we created a strain where LexA is deactivated (null-lexA) and measured the lexA promoter activity through the fluorescence of cerulean. Unexpectedly, $\alpha_{r}$ varied substantially between the promoter variants. It was largest for $P_{\text {lexA }}$ [MFI $(7.44 \pm 0.25) \times 10^{3}$ a.u.], less for $P_{\text {lexA-SOS5 }}\left[\mathrm{MFI}(6.28 \pm 0.12) \times 10^{3}\right.$ ] and $P_{\text {trc }}$-induced [MFI $\left.(5.19 \pm 0.38) \times 10^{3}\right]$, and least for $P_{t r c}$ [MFI $\left.(3.29 \pm 0.16) \times 10^{3}\right]$. Multiple experiments suggested that $P_{\text {lexA-SOS3 }}\left[\right.$ MFI $(1.02 \pm 0.06) \times 10^{4}$; Fig. S.4, [18]] is too unstable for us to make meaningful conclusions from this variant. These differences in $\alpha_{r}$ may influence mutational robustness.

By fitting a Hill function to the plots of fluorescence data against ATc, we can estimate the cooperativity of each promoter construct (Figs. S.3 and S.4 in the Supplemental Material [18]). The calculated Hill coefficient observed $\left(h_{\mathrm{obs}}\right)$ for $P_{\text {lexA }}$ of $1.5 \pm 0.2$ in this system agrees with previous estimates of LexA cooperativity ranging from 1 to $2.4[17,36,38]$. Surprisingly, addition of three SOS boxes
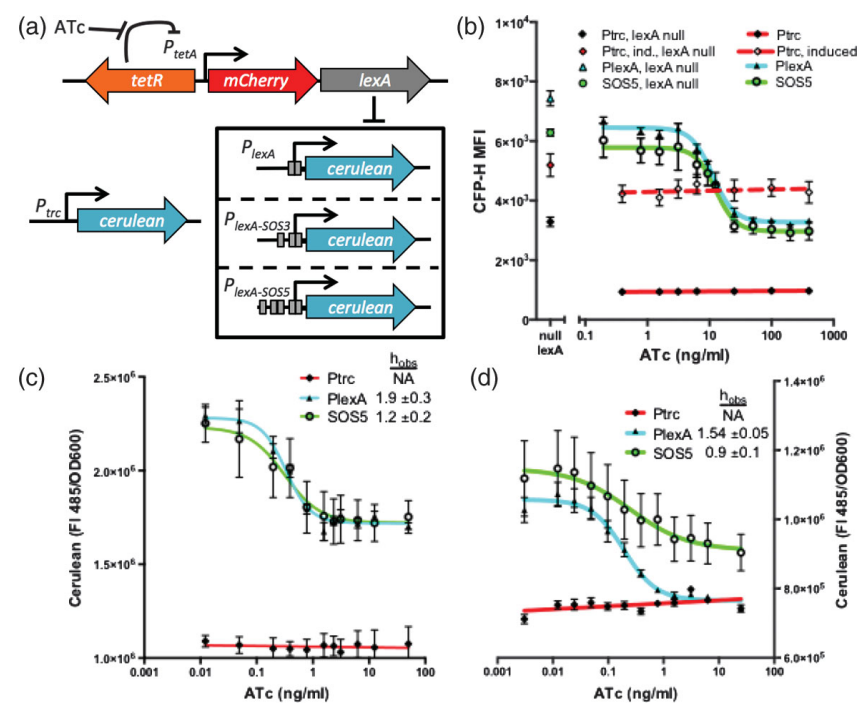

FIG. 3. Experimental modification and characterization of the Hill function. (a) Schematic of the genetic system with TetR control of lexA. (b) ATc dose-dependent transcription from $P_{t r c}$, $P_{\text {lexA }}$, and $P_{\text {lexA-SOS5 }}$ as inferred from cerulean fluorescence during flow cytometry. Symbols next to the $y$ axis indicate promoter activity in a null-lexA strain. $P_{\text {lexA }}$ and $P_{\text {lexA-SOS5 }}$ were grown in rich media and 30ng/mL MMC (mitomycin $\mathrm{C}$, which induces the cleavage of LexA); median fluorescence intensity (MFI). (c),(d) ATc dose-dependent cerulean fluorescence measured in a plate reader in the absence (d) or presence of 120ng/mL MMC (c). Panels (b)-(d) taken together show that the Hill coefficient in $P_{\text {lexA }}$ is greater than in $P_{\text {lexA-SOS5 }}$.

upstream of the RNA polymerase binding site in the $P_{\text {lexA-SOS5 }}$ construct had the effect of lowering $h_{\mathrm{obs}}$ to $1.2 \pm 0.2$. However, the ATc-inducible system may fall short of characterizing the Hill function at low LexA levels. In theory, the cerulean fluorescence levels (CFP-H) obtained in the null-lexA strain should be nearly equivalent to fluorescence levels observed at low ATc concentrations wherein LexA expression is fully repressed by TetR. However, cerulean levels in the null-lexA strain are actually 2 to 4 times higher than observed when TetR is fully repressing LexA expression in the absence of ATc. Therefore, we sought to further reduce LexA levels in the ATc-inducible system by activating a LexA coprotease, RecA, with mitomycin C [39]. By plotting fluorescence data against ATc under different growth conditions [Figs. 3(b)-(d)], we sample different ranges of LexA levels with Fig. 3(b) being at the lower end and Fig. 3(d) being at the higher end. We interpret the crossing between the Hill curves as a difference in the Hill coefficient. Overall, we find both $\alpha_{r}$ and $h_{\text {obs }}$ to be reduced in $P_{\text {lexA-SOS5 }}$ relative to $P_{\text {lexA }}$ in multiple assay conditions. This provides us the means to test the hypothesis that feedback strength, as determined by $\alpha_{r}$ and $h_{\text {obs }}$, influences mutational robustness.

In order to determine how the Hill function influences mutational robustness of the LexA gene circuit, we generated a panel of plasmids that place lexA-mCherry under control of 
either "no feedback" $\left(P_{t r c}\right)$, "NAR weak" $\left(P_{\text {lexA-SOS5 }}\right)$ or "NAR strong" $\left(P_{\text {lexA }}\right)$ [Fig. 4(a)]. The presence of mCherry downstream of lexA permits inference of promoter activity through measurement of mCherry fluorescence levels. This plasmid panel was transformed into $E$. coli cells with a chromosomally encoded $P_{\text {sulA }}-g f p$ reporter that is repressed by LexA to allow assessment of LexA repressor function via monitoring of GFP fluorescence levels. Next, we progressively introduced specific mutations into the lexA gene that severely attenuate repressor function in the cell by increasing degradation rate $[17,40]$. Any one of these substitutions is highly deleterious and their combination would be expected to render the LexA function irrecoverable. We also generated a R64A single mutant in the DNA binding domain that renders LexA unable to repress gene expression in order to measure the maximal expression from the $P_{\text {lexA }}, P_{\text {lexA-Sos5 }}$, and $P_{\text {sula }}$ promoters.

NAR rescues the repressor function from deleterious mutations and feedback strength further enhances this rescue [Fig. 4(b) and Table S.2 [18]]. As expected, in the absence of NAR, the Q92W mutant greatly attenuates LexA's ability to repress $P_{\text {sulA }}-g f p$, and introducing additional deleterious mutations increases $P_{\text {sula }}$-gfp expression to the point of being nearly indistinguishable from the empty vector control system that is devoid of LexA [Fig. 4(b), $P_{t r c}$ ]. Although simply raising LexA expression levels in the absence of NAR
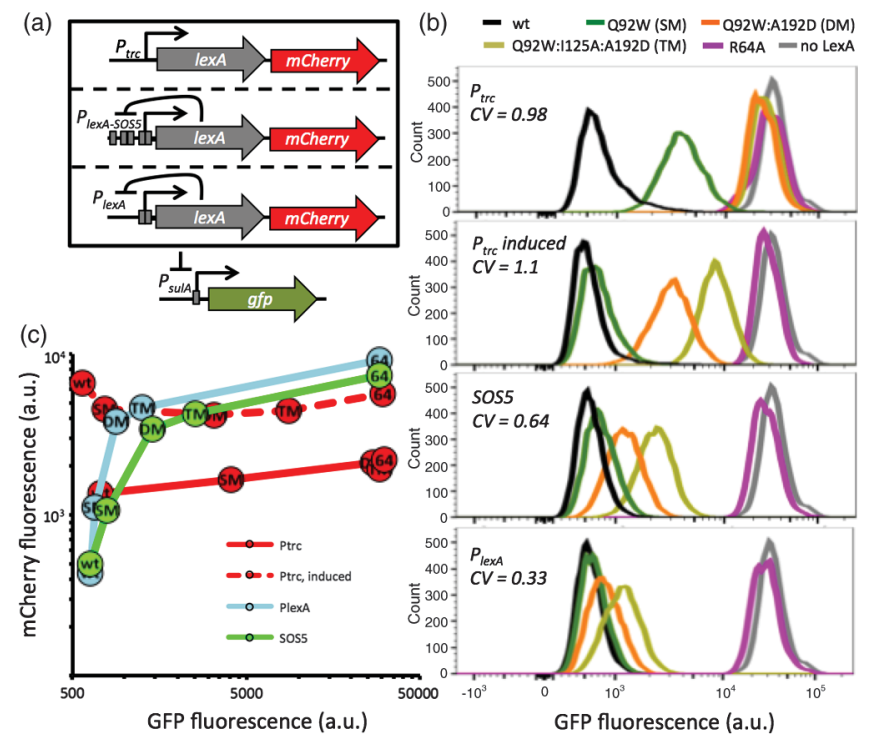

FIG. 4. Influence of feedback strength on LexA mutational robustness. (a) Schematic of the genetic system. (b) Representative flow cytometric data of GFP fluorescence distributions for each promoter construct. Coefficient of variation $(C V)$ using wt, $\mathrm{SM}, \mathrm{DM}$, and TM is taken as a measure of mutational robustness. (c) mCherry MFI is plotted against GFP MFI for each promoter construct. Each data point is the average MFI of either wt, Q92W (SM), Q92W:A192D (DM), Q92W:I125A:A192D (TM) or R64A (64) LexA taken across four biological replicates. Error bars representing the standard error of the mean are smaller than the data point labels. can rescue the Q92W mutant and lower GFP levels in others, the coefficient of variation $(C V)$ taken across wt, SM, DM, and TM mutants is not diminished [Figs. 4(b) $P_{t r c}$-induced and 2(d)]. This indicates that increasing $\alpha_{r}$ alone is not sufficient to significantly improve overall mutational robustness of this system. In the context of the "weak" negative feedback promoter $\left(P_{\text {lexA-SOS5 }}\right)$, these LexA mutants partially regain the cellular capacity to repress $P_{\text {sulA- }}$ gfp [Fig. 4(b) SOS5]. With stronger negative feedback, these mutants are further rescued [Fig. 4(b), $P_{\text {lexA }}$ ] and the $C V$ is even lower. Simultaneously monitoring mCherry fluorescence levels allows us to determine how each promoter responds to the introduction of the LexA mutations. As expected, mCherry levels are flat across the LexA mutants in the $P_{\text {trc }}$ construct lacking feedback [Fig. 4(c)]. In contrast, the feedback constructs are able to increase LexA expression in step with progressively deleterious mutations. These experimental data indicate a role for cooperativity in providing additional mutational robustness in target gene repression by increasing expression levels of the mutated transcriptional repressor.

Negative feedback TFs are more frequently mutated than other groups of genes.-In order to find further evidence for the influence of negative feedback on mutational robustness, we turned to the long-term experimental evolution project of the Richard Lenski lab. At 40000 generations of serial passaging [41], we found 16 out of 68 negative feedback TF genes [42] have nonsynonymous mutations while just 10 out of 93 TFs without negative feedback have these mutations $\left(\chi^{2}\right.$ test $\left.p=0.030\right)$. More broadly, across all 4290 genes, 621 of which are mutated, the 68 negative feedback TFs are more frequently mutated than expected $\left(\chi^{2}\right.$ test $\left.p=0.032\right)$. This suggests a role for negative feedback in shaping the mutational landscape of an evolving bacterial population.

Conclusion.-Negative feedback imparts robustness to changes in most system parameters, but not all [43]. Changing the strength of a repressor's self-repression $\left(K_{r}\right)$ results in a significant change of repressor protein level and higher cooperativity increases the sensitivity of repressor protein levels ( $r$ ) to changes in $K_{r}$ [Fig. 2(b)]. And yet, downstream gene repression still remains insulated against changes in $K_{r}$ so long as the repressor's affinity for the target promoter $\left(K_{g}\right)$ changes in step [Fig. 2(c)]. Changing both $K_{r}$ and $K_{g}$ simultaneously may be achieved by amino acid mutations in the repressor that directly affect DNA affinity. Mutations reducing DNA affinity result in elevated protein levels of the mutant repressor and repression of target promoters can be maintained because repressor concentration in the cell has shifted to match the change in $K_{g}$. However, mutations that specifically change $K_{r}$, such as a substitution in the DNA recognition site of the repressor's promoter, will elevate expression of a fully functional repressor and have a disproportionate repression effect on other genes whose affinities for the repressor are unchanged. Therefore, which parameter(s) a mutation alters determines how the negative feedback network responds. 
Previous theoretical and computational work noted the influence of cooperativity on NAR's suppression of variability. Using a power-law formalism and sensitivity analysis, it was shown that robustness to changes in the degradation rate is enhanced by NAR of regulator gene expression [44] and this robustness increases with kinetic order. This kinetic order corresponds to a Hill coefficient in the limit of strong regulator-promoter binding. Other work combining stochastic modeling with parameter variation likewise noted the effect of NAR cooperativity in buffering repressor levels against changes in degradation rate $[45,46]$. We build upon this work by showing that changes in $\beta_{r}$ and $K_{r}$ can have similar downstream effects [Fig. 2(c)] and specifically interpreting mutations as sources of long-lived (as opposed to transient) changes in these parameters. Then we continue by experimentally verifying the importance of cooperativity in maintaining stable expression of downstream genes in the presence of detrimental mutations to the repressor. Also, by simultaneously following LexA promoter output (mCherry) and target gene output (GFP), we experimentally show a concerted response to increasingly deleterious repressor mutations.

In eukaryotes, understanding the role of negative feedback loops in cell survival and evolution is critical because there are a large number of proteins whose expression is governed by negative feedback loops, including many TFs involved in cancer, such as p53. Defects in negative feedback mechanisms are capable of enhancing proliferative cell signaling that promotes tumorigenesis [47]. In fact, specific disruption of p53's negative feedback loop results in extreme radiosensitivity [48] and multiple clinical trials are underway that target p53 or its feedback loop [49]. Therefore, the emergence of this mechanism is broadly relevant to human health and to the pursuit of drugs that target negative feedback mechanisms.

The authors gratefully acknowledge support from the National Institutes of Health (GM066099 and GM079656 to O.L.; R01GM088653 to C. H.), from the National Science Foundation (DBI-1356569 to O.L.), and from DARPA (N66001-15-C-4042 to O.L.). This project was also supported by the Cytometry and Cell Sorting Core at Baylor College of Medicine with funding from the NIH (P30 AI036211, P30 CA125123, and S10 RR024574) and the expert assistance of Joel M. Sederstrom. We thank Dominik Satory for providing the DS140 strain. We thank Ido Golding and Herman Dierick for helpful suggestions on the manuscript.

D. C. M. and R. C. L contributed equally to this work.

*Corresponding author.

lichtarge@bcm.edu

[1] J. Masel and M. L. Siegal, Trends Genet. 25, 395 (2009).
[2] L. Zheng, L. A. Sepúlveda, R. C. Lua, O. Lichtarge, I. Golding, and A. M. Sokac, PLoS Genet. 9, e1003901 (2013).

[3] S. L. Rutherford and S. Lindquist, Nature (London) 396, 336 (1998).

[4] D. F. Jarosz and S. Lindquist, Science 330, 1820 (2010).

[5] C. Queitsch, K. D. Carlson, and S. Girirajan, PLoS Genet. 8, e1003041 (2012).

[6] J. Lempe, J. Lachowiec, A. M. Sullivan, and C. Queitsch, Curr. Opin. Plant Biol. 16, 62 (2013).

[7] A. Steinacher, D. G. Bates, O. E. Akman, and O. S. Soyer, PLoS One 11, e0153295 (2016).

[8] D. Madar, E. Dekel, A. Bren, and U. Alon, BMC Syst. Biol. 5, 111 (2011).

[9] M. A. Savageau, Nature (London) 252, 546 (1974).

[10] N. Rosenfeld, M. B. Elowitz, and U. Alon, J. Mol. Biol. 323, 785 (2002).

[11] F. M. Camas, J. Blazquez, and J. F. Poyatos, Proc. Natl. Acad. Sci. U.S.A. 103, 12718 (2006).

[12] A. Becskei and L. Serrano, Nature (London) 405, 590 (2000).

[13] M. Thattai and A. van Oudenaarden, Proc. Natl. Acad. Sci. U.S.A. 98, 8614 (2001).

[14] D. Nevozhay, R. M. Adams, K. F. Murphy, K. Josic, and G. Balazsi, Proc. Natl. Acad. Sci. U.S.A. 106, 5123 (2009).

[15] C. M. Denby, J. H. Im, R. C. Yu, C. G. Pesce, and R. B. Brem, Proc. Natl. Acad. Sci. U.S.A. 109, 3874 (2012).

[16] O. P. Gross, E. N. Pugh, and M. E. Burns, Neuron 76, 370 (2012).

[17] D. C. Marciano, R. C. Lua, P. Katsonis, S. R. Amin, C. Herman, and O. Lichtarge, Cell Rep. 7, 1789 (2014).

[18] See Supplemental Material at http://link.aps.org/ supplemental/10.1103/PhysRevLett.116.258104 for additional analysis of the model and data, and experimental materials and methods, which includes Refs. [19-34].

[19] M. A. Savageau, Nature (London) 229, 542 (1971).

[20] A. Goldbeter and D. E. Koshland, Proc. Natl. Acad. Sci. U.S.A. 78, 6840 (1981).

[21] S. Ramsey, D. Orrell, and H. Bolouri, J. Bioinform. Comput. Biol. 03, 415 (2005).

[22] J. Quan and J. Tian, Nat. Protoc. 6, 242 (2011).

[23] S. N. Ho, H. D. Hunt, R. M. Horton, J. K. Pullen, and L. R. Pease, Gene 77, 51 (1989).

[24] W. Jiang, D. Bikard, D. Cox, F. Zhang, and L. A. Marraffini, Nat. Biotechnol. 31, 233 (2013).

[25] P. P. Cherepanov and W. Wackernagel, Gene 158, 9 (1995).

[26] O. Lichtarge, H. R. Bourne, and F. E. Cohen, J. Mol. Biol. 257, 342 (1996).

[27] S. Madabushi, H. Yao, M. Marsh, D. M Kristensen, A. Philippi, M. E Sowa, and O. Lichtarge, J. Mol. Biol. 316, 139 (2002).

[28] I. Mihalek, I. Res, and O. Lichtarge, J. Mol. Biol. 336, 1265 (2004).

[29] A. D. Wilkins, E. Venner, D. C. Marciano, S. Erdin, B. Atri, R. C. Lua, and O. Lichtarge, Bioinformatics 29, 2714 (2013).

[30] R. S. Cox, M. J. Dunlop, and M. B. Elowitz, J. Biol. Eng. 4, 10 (2010).

[31] S. Warming, N. Costantino, D. L. Court, N. A. Jenkins, and N. G. Copeland, Nucleic Acids Res. 33, e36 (2005).

[32] K. A. Datsenko and B. L. Wanner, Proc. Natl. Acad. Sci. U.S.A. 97, 6640 (2000). 
[33] T. Baba, T. Ara, M. Hasegawa, Y. Takai, Y. Okumura, M. Baba, K. A. Datsenko, M. Tomita, B. L. Wanner, and H. Mori, Mol. Syst. Biol. 2, 2006.0008 (2006).

[34] J. M. Pennington and S. M. Rosenberg, Nat. Genet. 39, 797 (2007).

[35] U. Alon, An Introduction to Systems Biology: Design Principles of Biological Circuits (Chapman and Hall/ CRC, London, 2006).

[36] M. Ronen, R. Rosenberg, B. I. Shraiman, and U. Alon, Proc. Natl. Acad. Sci. U.S.A. 99, 10555 (2002).

[37] E. C. Friedberg, G. C. Walker, W. Siede, R. D. Wood, R. A. Schultz, and T. Ellenberger, DNA Repair and Mutagenesis (ASM Press, Washington, DC, 2006).

[38] S. V. Aksenov, J. Biol. Phys. 25, 263 (1999).

[39] A. K. Adikesavan, P. Katsonis, D. C. Marciano, R. Lua, C. Herman, and O. Lichtarge, PLoS Genet. 7, e1002244 (2011).
[40] M. H. Smith, M. M. Cavenagh, and J. W. Little, Proc. Natl. Acad. Sci. U.S.A. 88, 7356 (1991).

[41] J. E. Barrick and R. E. Lenski, Cold Spring Harbor Symp. Quant. Biol. 74, 119 (2009).

[42] T. Warnecke, G. Z. Wang, M. J. Lercher, and L. D. Hurst, BMC Evol. Biol. 9, 193 (2009).

[43] W. S. Hlavacek and M. A. Savageau, J. Mol. Biol. 248, 739 (1995).

[44] M. E. Wall, W. S. Hlavacek, and M. A. Savageau, J. Mol. Biol. 332, 861 (2003).

[45] T. Toni and B. Tidor, PLoS Comput. Biol. 9, e1002960 (2013).

[46] A. Singh and J. P. Hespanha, Biophys. J. 96, 4013 (2009).

[47] D. Hanahan and R. A. Weinberg, Cell 144, 646 (2011).

[48] V. Pant, S. Xiong, J. G. Jackson, S. M. Post, H. A. Abbas, A. Quintás-Cardama, A. N. Hamir, and G. Lozano, Genes Dev. 27, 1857 (2013).

[49] C. F. Cheok, C. S. Verma, J. Baselga, and D. P. Lane, Nat. Rev. Clin. Oncol. 8, 25 (2011). 